poetry and ideas from the history of the longest continuous civilization on Earth. The relationship of that civilization with its fragile and often tortured surroundings contains lessons for others - particularly at a time when industrial society in China, as elsewhere, is pressing harder than ever on the environment. This will be a source book, elephants and all, for generations to come. Crispin Tickell is a member of the China Council for International Cooperation on Environment and Development. \section{The body-plan explosion}

(1)

\section{On the Origin of Phyla}

by James W. Valentine

University of Chicago Press: 2004. 614 pp.

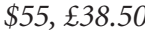

\section{Stefan Bengtson}

More than 30 years ago, palaeontologist James W. Valentine's highly acclaimed book Evolutionary Paleoecology of the Marine Biosphere sought to integrate ecological and environmental studies within a framework of evolution over geological time. He has now written a book on one of the most significant revolutions in the history of life, the Cambrian explosion.

The Cambrian explosion is the transition half-a-billion years ago from a fundamentally microbial biosphere to one in which various multicellular creatures expanded the food web to dimensions and shapes never before realized. It saw the appearance of just about all the major groups of animals that we today recognize as (fossilizable) phyla. Yet Valentine's new book of more than 600 pages contains little about ecology and almost nothing about the physical environment. What has changed since 1973 ?

For one thing, Valentine, one of the renaissance minds of our times, has further expanded his vision to take in new fields of science, notably developmental genetics, molecular systematics and the evolutionary development (evo-devo) melting pot. Even so, the decision to downplay environmental aspects of early animal evolution must be understood as a deliberate one.

Darwin wisely called his best-known work On The Origin of Species; the origin of phyla is an even stickier problem, and Valentine deserves credit for tackling it at such breadth. The first problem is defining the concept of the phylum in an evolutionary context. Historically, phyla have been recognized as groups of extant animals with a characteristic body plan and uncertain relationships to other such groups. Fossils have generally been assigned to the least dissimilar of the living phyla, a practice that pre-empts

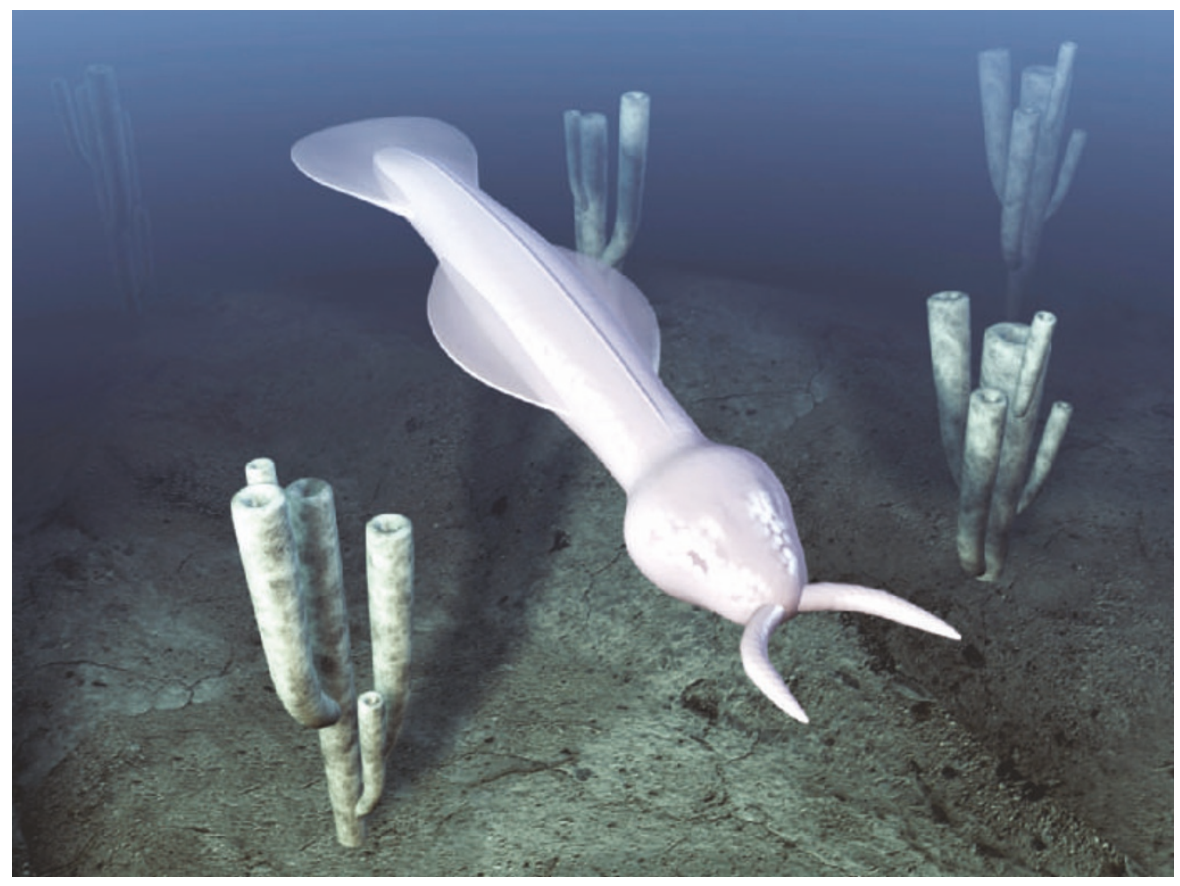

In a phylum of its own? Fossils of Amiskwia suggest that it was unlike any other known animal.

the possibility of extinct phyla. From a cladistic point of view, it is easy to argue that two extant sister phyla arose at the point of branching from the last common ancestor, and that anything that branched off later should be included in the respective phylum. A distinction can then be made between the crown group (the descendants of the last common ancestor of all the living members of the phylum, including this ancestor) and the stem group (everything else). This avoids the question of how body plans arise and whether there may be others not represented by living forms.

Defining a body plan isn't easy, however. Valentine's definition, for example, is dangerously circular: "an assemblage of morphological features shared among members of a phylum-level group". What does that mean, except that when we define a phylum we also define its body plan, or vice versa? Valentine proposes to define the origin of a phylum by the acquisition of a key apomorphy - a unique derived trait. This may be more subjective and less convenient than letting the total (stem and crown) group or the crown group define the phylum, but it gives due priority to biological significance over methodological convenience. After all, we want to know how different kinds of organism evolve by natural selection, and how they interact with each other and with the environment. They do that with their phenotypes, not their pedigrees.

A key question, then, is whether the body plans of the recognized phyla represent more or less the total number of possible solutions to the problem of being an animal, or whether there were numerous other possibilities that came into being but became extinct because of bad luck or bad design.
Valentine argues that the Cambrian explosion initially produced great disparity in design, but that this was subsequently diminished by extinctions. The pattern of diminishing evolutionary novelty subsequent to this event, he says, may have been due less to developmental constraints than to a saturation effect (candidates for new adaptive radiations were already available among existing body plans). He also believes that the Cambrian explosion produced a lot more homoplasies (similar characters with independent origins) than most phylogenetic analyses suggest - in my view an extremely important point that calls for much more careful character evaluation than is commonly done. He is clearly not impressed, then, by some recent attempts to use fossils to bridge gaps between phyla.

Valentine seems most happy with intrinsic biological mechanisms for the rapid appearance of phyla. Large parts of the book deal with developmental prerequisites (such as cell-type numbers and gene regulation) for the event. Ecological interactions, such as predation, are given more cursory treatment. As for the physical environment, he merely concludes, somewhat apologetically, that although physical environmental factors were "supremely important", he does not see any evidence that extraordinary environmental events were causally connected with the Cambrian explosion. Given that extraordinary environmental events did indeed occur shortly before the explosion, I would give the jury just a little more time to ponder the question. But first I would make sure they had read this magnificent book.

Stefan Bengtson is in the Department of Palaeozoology, Swedish Museum of Natural History, Box 50007, SE-10405 Stockholm, Sweden. 